\title{
Topological quantum phase transition between Fermi liquid phases in an Anderson impurity model
}

\author{
G. G. Blesio and L. O. Manuel \\ Instituto de Física Rosario (CONICET) and Universidad Nacional de Rosario, \\ Bv. 27 de Febrero 210 bis, 2000 Rosario, Argentina \\ P. Roura-Bas and A. A. Aligia \\ Centro Atómico Bariloche and Instituto Balseiro, \\ Comisión Nacional de Energía Atómica, CONICET, 8400 Bariloche, Argentina
}

\begin{abstract}
We study a generalized Anderson model that mixes two localized configurations -one formed by two degenerate doublets and the other by a triplet with single-ion anisotropy $D S_{z}^{2}-$ by means of two degenerate conduction channels. The model has been derived for a single Ni impurity embedded into an $\mathrm{O}$-doped $\mathrm{Au}$ chain. Using the numerical renormalization group, we find a topological quantum phase transition, at a finite value $D_{c}$, between two regular Fermi liquid phases of high (low) conductance and topological number $2 I_{L} / \pi=0(-1)$ for $D<D_{c}\left(D>D_{c}\right)$, where $I_{L}$ is the well-known Luttinger integral. At finite temperature the two phases are separated by a non-Fermi liquid phase with fractional impurity entropy $\frac{1}{2} \ln 2$ and other properties which remind those of the two-channel Kondo model.
\end{abstract}

PACS numbers: 71.10.Hf, 73.63.-b, 72.15.Qm

Introduction- Quantum phase transitions (QPTs) observed in transport through molecular systems in which two electrons play a relevant role have been a subject of interest recently [1 $[3]$. In general, in nanoscopic systems with more than one electron, the spin-orbit coupling is important and leads to the single-ion anisotropy $D S_{z}^{2}$, where $S_{z}$ is the total spin of the molecule or quantum dot $2[5,[8]$. The relative magnitude of $D$ can be tuned experimentally [2, 6, 8]. On the other hand, experiments with mechanically controllable break junctions made possible to create one-dimensional atomic chains of several elements, and measure the conductance through them [9, 10].

Some QPTs are topological QPTs (TQPTs): even if some other properties vary continuously at the transition, a topological integer (related with a geometrical Berry phase or the topology of each thermodynamic phase) jumps at the TQPT. Examples of this kind of transitions are several charge and spin TQPTs observed in onedimensional models in which the nearest-neighbor hopping depends on the occupation [11, 12] as in cold-atom lattices [13], or the Hubbard model with alternative onsite energies 14, 15], for which the topological transition might be observable in transport through arrays of quantum dots or molecules [16].

On the other hand, in condensed matter physics, the Luttinger theorem [17, 18], which states that the volume of the Fermi surface is determined by the particle density and remains unaltered by interactions, and Friedel sum rules [19, 20], which relate the occupancy of impurity states with the corresponding spectral density at the Fermi energy, have been crucial for our present understanding of many interacting systems that behave as Fermi liquids at zero temperature. The so-called Lut- tinger integral $I_{L}$ 21] enters the demonstrations in these works and it was generally assumed to vanish. However, recently a group of researchers found that $I_{L}$ can take three different values in an impurity model in phases with regular low-energy Fermi liquid behavior [22, 23]. This is surprising since only $I_{L}=0$ was expected in a regular Fermi liquid, according to its perturbative calculation in the seminal work by Luttinger and Ward [18], where the Fermi liquid was considered adiabatically connected to a system of non-interacting electrons. More recently, Seki and Yunoki 24] showed that the Luttinger integral, which is the deviation of the Luttinger volume from the non-interacting limit, can be interpreted as a winding number of the ratio between the determinants of the noninteracting and interacting single-particle Green's functions. The combination of this topological interpretation of $I_{L}$ and the finding of its non-zero values 22, 23] opens the possibility of topologically non-trivial Fermi liquid phases, that are not adiabatically connected with noninteracting systems and, therefore, they can be termed as non-Landau Fermi liquids.

In this Letter, we describe the TQPT that, driven by the single-ion anisotropy $D$, takes place in a simple impurity model which, in the Kondo limit, consists in a spin 1 screened by two conduction channels. This model has been derived from $a b$ initio calculations and describes transport through $\mathrm{Ni}$ atoms in $\mathrm{O}$ doped $\mathrm{Au}$ chains 25, 26]. The oxygen doping has the effect of pushing up the $5 d_{x z}$ and $5 d_{y z}$ bands of $\mathrm{Au}$ (with $z$ along the chain direction), which are below the Fermi energy in pure $\mathrm{Au}$ chains [26 28]. The two conduction channels correspond to the degenerate orbitals of $x z$ and $y z$ symmetry, and they hybridize with the corresponding Ni $3 d$ orbitals. Solving the model by means of the numerical renormal- 
ization group (NRG), we find that the transition occurs at the finite critical anisotropy $D_{c} \approx 2.57 T_{K}^{0}$, where $T_{K}^{0}$ is the Kondo temperature for $D=0$. This TQPT separates two regular Fermi liquid phases: for $D<D_{c}$ the impurity spin is Kondo screened, while for $D>D_{c}$ it is quenched by the anisotropy. For $D<D_{c}$, as the temperature $T \rightarrow 0$, the electrical conductance is large and agrees with the usual Friedel sum rule with $I_{L}=0$, as in Fermi liquids adiabatically connected with a noninteracting system. Instead, for $D>D_{c}$ and $T=0$, the conductance is small and satisfies a generalized Friedel sum rule with $I_{L}=-\frac{\pi}{2}$, corresponding to a non-Landau Fermi liquid. Furthermore, for $D \approx D_{c}$ and in a finite interval of temperatures $T^{*}(D) \leq T \lesssim T_{K}^{0}$, where $T^{*}(D) \rightarrow 0$ as $D \rightarrow D_{c}$ [29], there is a critical quantum regime whose electrical transport and thermodynamics properties correspond to a non-Fermi liquid behavior. It is worth to mention that $T_{K}^{0}$ can be tuned by stretching the gold chains, rendering it possible to observe the transition experimentally.

Model- We consider the Hamiltonian that describes a system containing a $\mathrm{Ni}$ atom in a substitutional position within a Au chain doped with a small amount of oxygen $(\sim 14 \%)$ [25, 26], describing charge fluctuations between $3 d^{8}$ and $3 d^{9} \mathrm{Ni}$ configurations. It can be written as 30 ]

$$
\begin{aligned}
H & =\sum_{M_{2}}\left(E_{2}+D M_{2}^{2}\right)\left|M_{2}\right\rangle\left\langle M_{2}\left|+\sum_{\alpha M_{1}} E_{1}\right| \alpha M_{1}\right\rangle\left\langle\alpha M_{1}\right|+ \\
& +\sum_{\nu k \alpha \sigma} \varepsilon_{\nu k} c_{\nu k \alpha \sigma}^{\dagger} c_{\nu k \alpha \sigma}+ \\
& +\sum_{\substack{M_{1} M_{2} \\
\alpha \nu k \sigma}} V_{\nu}\left\langle 1 M_{2} \mid \frac{1}{2} \frac{1}{2} M_{1} \sigma\right\rangle\left(\left|M_{2}\right\rangle\left\langle\alpha M_{1}\right| c_{\nu k \alpha \sigma}+\text { H.c. }\right)
\end{aligned}
$$

where $E_{i}$ and $M_{i}$ indicate the energies and the spin projections along the chain, respectively, of states with $i=$ 1,2 holes in the $3 d$ shell of the Ni impurity; $\left|\alpha M_{1}\right\rangle$ is the state with one hole with symmetry $\alpha(x z, y z)$ and spin $M_{1}$. $D$ is the Ni uniaxial magnetic anisotropy. The operator $c_{\nu k \alpha \sigma}^{\dagger}$ creates a hole with symmetry $\alpha$ and energy $\varepsilon_{\nu k}$ (relative to the Fermi level $\varepsilon_{F}=0$ ) in the $5 d$ shell of the Au atom, where $\nu=L, R$ denotes the left or the right side of the Ni atom, respectively. $\left\langle 1 M_{2} \mid \frac{1}{2} \frac{1}{2} M_{1} \sigma\right\rangle$ are ClebshGordan coefficients. The hopping $V_{\nu}$ characterizes the tunneling between the $\mathrm{Ni}$ and $\mathrm{Au}$ states, and it enters the hybridization function $\Delta=\pi \sum_{\nu k}\left|V_{\nu}\right|^{2} \delta\left(\omega-\varepsilon_{\nu k}\right)$, assumed independent of energy.

The ground-sate configuration of the $\mathrm{Ni}$ atom has two holes in the degenerate $3 d_{x z}, 3 d_{y z}$ orbitals coupled to spin $S=1$. The state $\left|M_{2}\right\rangle$ with $M_{2}=0$ is lower in energy than those with $M_{2}= \pm 1$ by an energy that has been estimated in $D \approx 8.5 \mathrm{meV}$ solving exactly the atomic model for the $3 \mathrm{~d}^{8}$ configuration including all interactions and spin-orbit coupling 25].

We solve the Hamiltonian (1) by means of NRG, as implemented in the Ljubljana open source code [31]. We use a discretization parameter $\Lambda=3$, and we keep up to 10000 states. The results are $z$-averaged with $N_{z}$ up to 4. In this work, we have chosen $\varepsilon_{d} \equiv E_{1}-E_{2}=-0.02$ and $\Delta=0.1$ in units of the conduction half-bandwidth $W$. For this ratio $\varepsilon_{d} / \Delta$, the system is in the Kondo regime, but close to the mixed valence regime (MVR), and the different regimes that we want to display come out more clearly. The corresponding Kondo temperature is $T_{K}^{0} \simeq 1.245 \times 10^{-3}$ for $D=0$, obtained through the usual condition $G\left(T_{K}^{0}\right)=\frac{1}{2} G(T \rightarrow 0)$, where $G(T)$ is the differential conductance $G=d I / d V$. However, the occupancy found in ab-initio calculations [25, 26] indicates that the system is closer to the MVR and with a Kondo temperature near $6 \mathrm{meV}$, as explained in the Supplemental Material [30]. The corresponding value $D_{c} \simeq 2.57 T_{K}^{0} \sim 15 \mathrm{meV}$ roughly falls in the range of the estimated $D$ for $\mathrm{Ni}$ atoms in $\mathrm{O}$ doped $\mathrm{Au}$ chains, particularly taking into account that $D$ can be reduced by stretching or tuned by further doping the gold chains.

Generalized Friedel sum rule- Using conservation laws, the impurity spectral function per orbital and spin, at the Fermi level and $T=0$, is given by [20]

$$
A_{d \alpha \sigma}(\omega=0)=\frac{1}{\pi \Delta} \sin ^{2}\left(\delta_{\alpha \sigma}\right),
$$

where, taking into account explicitly the spin degeneracy, the phase shift is

$$
\delta_{\alpha \sigma}=\frac{\pi}{2}\left\langle n_{d \alpha}\right\rangle-I_{L} .
$$

$n_{d \alpha}=\sum_{M_{2}}\left|M_{2}><M_{2}\right|+\sum_{M_{1}}\left|\alpha M_{1}><\alpha M_{1}\right|$ is the hole occupation number of the Ni $\alpha$ orbital, and the Luttinger integral $I_{L}$, which in our case is independent of orbital and spin indices, is defined as

$$
I_{L}=\operatorname{Im} \int_{-\infty}^{0} d \omega G_{d \alpha \sigma}(\omega) \frac{\partial \Sigma_{d \alpha \sigma}(\omega)}{\partial \omega},
$$

where $G_{d \alpha \sigma}(\omega)$ is the impurity Green function for orbital $\alpha$ and spin $\sigma$ and $\Sigma_{d \alpha \sigma}(\omega)$ is the corresponding self energy.

As explained above, $I_{L}$ vanishes for a Fermi liquid, when it is perturbatively calculated from a noninteracting electronic system [18]. However, recently 22, 23] it was found that this is not always the case for local Fermi liquids, while a topological interpretation of $I_{L}$ was provided for extended systems 24].

$N R G$ results- To localize the TQPT we use the differential conductance, which is easily accessible experimentally [9, 10]. The conductance per channel is given by

$$
G_{\alpha}(T)=G_{0} \sum_{\sigma} \frac{\pi \Delta}{2} \int d \omega\left(-\frac{\partial f(\omega)}{\partial \omega}\right) A_{d \alpha \sigma}(\omega),
$$

where $f(\omega)$ is the Fermi function and $G_{0}=2 e^{2} / h$ is the quantum of conductance. Using Eqs. (2) and (3) we 
have, at zero temperature, the generalized Friedel sum rule for the conductance

$$
G_{\alpha}(0)=G_{0} \sin ^{2}\left(\frac{\pi}{2}\left\langle n_{d \alpha}\right\rangle-I_{L}\right) .
$$

In Fig. 11 the conductance per channel as a function of $T / T_{K}^{0}$, for several positive values of the singleion anisotropy $D$ is shown. $G_{\alpha}$ has an abrupt change as $D$ is varied across its critical value $D_{c} \simeq 0.003196$, and two regimes are easily characterized according to the behavior of $G_{\alpha}$ at the lowest temperatures. For $D<D_{c}$, $G_{\alpha}(T \rightarrow 0)$ takes a large value, which corresponds to the Friedel sum rule (6) with $I_{L}=0$. In this case, for low temperatures we expect a fully Kondo screened impurity, leading to the usual Fermi liquid phase. Note that, as we are working far away from the particle-hole symmetric point (only $3 d^{8}$ and $3 d^{9} \mathrm{Ni}$ configurations are considered), the occupation number per impurity orbital is less than $1\left(\left\langle n_{d \alpha}\right\rangle \simeq 0.788\right.$, almost constant with varying $\left.D\right)$ and $G_{\alpha}$ does not reach the unitary limit. It can be seen that, for small $D$, the conductance exhibits fingerprints of the magnetic anisotropy for temperatures of the order of $D$, like the shoulder that develops before it goes to its Kondo limit with decreasing $T$.

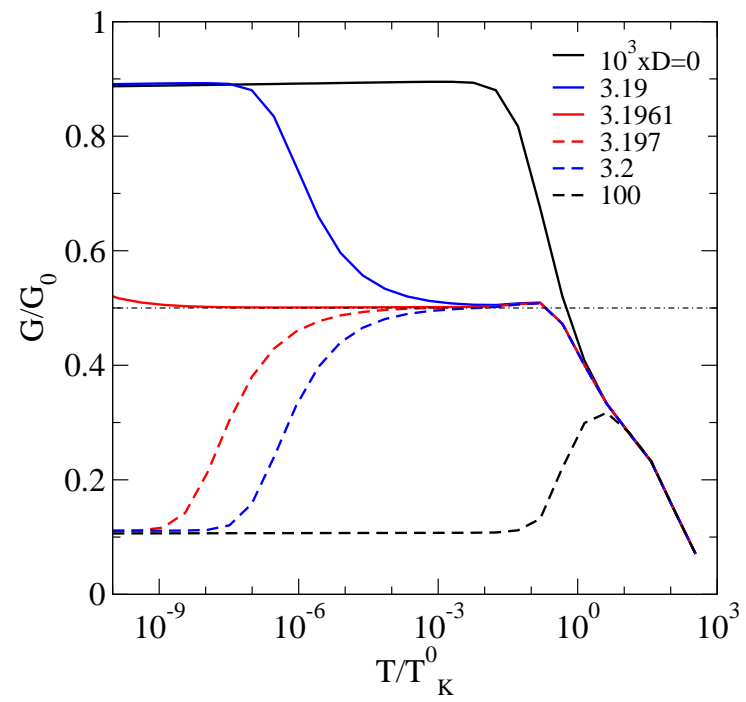

FIG. 1: (Color online) Electrical differential conductance as a function of $T / T_{K}^{0}$ for several values of the single-ion anisotropy $D$.

On the other hand, for $D>D_{c}, G_{\alpha}(T \rightarrow 0)$ goes to a low value, corresponding to $I_{L}=-\frac{\pi}{2}$ in (6). This case corresponds to the impurity spin quenched by $D$, as its ground state has spin projection $S_{z}=0$. Again, we expect a Fermi liquid at low $T$, but now this phase yields the non-trivial Luttinger integral $I_{L}=-\frac{\pi}{2}$. For $D \gg D_{c}, G_{\alpha}$ takes small values for any temperature.

For $D$ close to $D_{c}$, below and above, the conductance has a clear plateau at the precise value $G_{0} / 2$, characteristic of the two-channel Kondo (2CK) effect.
Around $D_{c}$ we define a characteristic energy for each phase. For $D<D_{c}, T_{K}^{*}(D)$ is computed through the condition $G_{\alpha}\left(T_{K}^{*}(D)\right)=\left(G_{\alpha}(T \rightarrow 0)-0.5 G_{0}\right) / 2$, corresponding to the onset of the fully Kondo screening of the impurity, while for $D>D_{c}$, we take $T_{q}^{*}(D)$ which satisfies $G_{\alpha}\left(T_{q}^{*}(D)\right)=\left(0.5 G_{0}-G_{\alpha}(T \rightarrow 0)\right) / 2$, and it signals the onset of the impurity spin quenching. We find that, as it corresponds to a quantum critical point [29], these energies vanish as $D \rightarrow D_{c}$. Surprisingly for a Kondo screening energy scale, $T_{K}^{*}(D)$ has a potential law dependence on $D$ :

$$
T_{K}^{*}(D) \propto T_{K}^{0}\left(\frac{D_{c}-D}{D_{c}}\right)^{2} .
$$

On the other hand,

$$
T_{q}^{*}(D) \propto T_{K}^{0} \exp \left[-c\left(\frac{T_{K}^{0}}{D-D_{c}}\right)^{1 / 4}\right],
$$

where $c$ is a constant of order of one; a similar result was fous

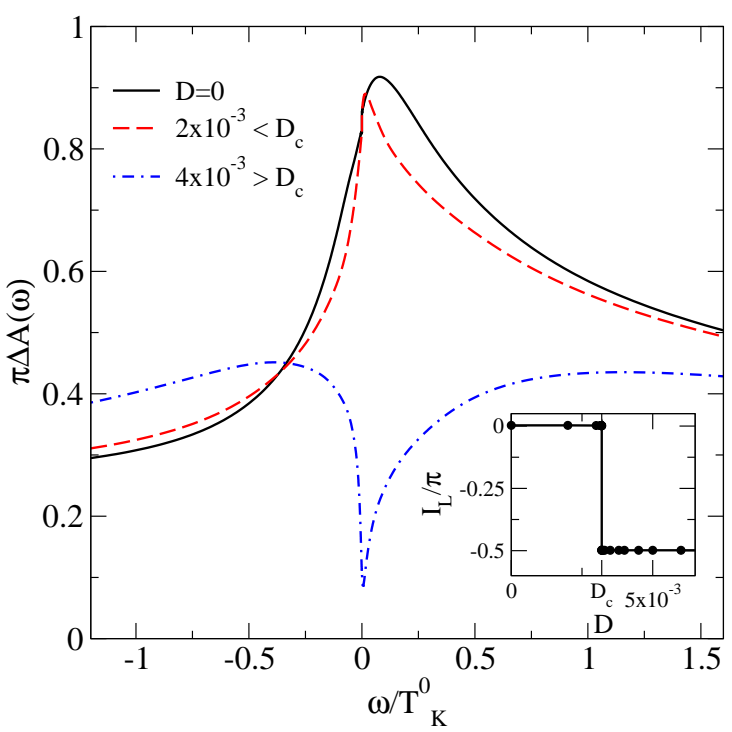

FIG. 2: (Color online) Impurity spectral function $A_{d \alpha \sigma}(\omega)$ for three different anisotropies $D$, at $T \simeq 10^{-6} T_{K}^{0}$. Inset: Luttinger integral $I_{L}$ as a function of $D$.

The impurity spectral function $A_{d \alpha \sigma}(\omega)$ near the Fermi level is presented in the main panel of Fig. 2, for three different values of $D$, at the very low temperature $T \simeq 10^{-6} T_{K}^{0}$. A Kondo resonance is clearly visible for all $D<D_{c}$. As $D$ increases, the Kondo peak moves towards the Fermi level and its width decreases. At $D_{c}$ the resonance abruptly disappears, and it is replaced by a narrow dip just at $\omega=0$. In the supplemental material [30], we show that for the related $S=1$ Kondo impurity model with two conduction channels, very close to its critical point, the spectral function (defined through the 
$t$-matrix) takes half of its Kondo-screened value, being this another hallmark of the $2 \mathrm{CK}$.

With the NRG technique it is not an easy task to obtain reliable values of $I_{L}$ by computing it directly from Eq. (4), due to numerical inaccuracies in the self-energy evaluation 22]. Instead, we calculate $I_{L}$ through the generalized Friedel sum rule for the conductance (6). The obtained $I_{L}$ is displayed as a function of $D$ in the inset of Fig. 2, It can be seen that $I_{L}$ takes only two discrete values: $I_{L}=0,-\pi / 2$, with an abrupt jump at $D_{c}$. This is not fortuitous as $I_{L}$ is closely related with the winding number of the ratio $D_{d}(z)=G_{d \alpha \sigma}^{0}(z) / G_{d \alpha \sigma}(z)$ between the non-interacting and interacting impurity Green's functions, around the origin in the complex plane $D_{d}$ (see supplemental material [30]):

$$
I_{L}=\pi \lim _{T \rightarrow 0} \oint_{\Gamma} \frac{d z}{2 \pi i} n_{F}(z) \frac{\partial \ln D_{d}(z)}{\partial z},
$$

where the contour $\Gamma$ encloses the real axis. So, the two Fermi liquids for $D \lessgtr D_{c}$ can be topologically distinguished by $I_{L}$, being topologically trivial (nontrivial) synonymous of adiabatically (non-adiabatically) connected to a non-interacting system. As a consequence, the quantum critical transition between the two Fermi liquids (a Landau- and a non-Landau Fermi liquid) at $D_{c}$ has a topological character. With the necessary caution due to the difficulties of the NRG computation of the self-energy [33], we have checked that its imaginary part $\operatorname{Im} \Sigma_{d \alpha \sigma}(\omega)$ behaves quadratically as a function of frequency close to the Fermi level, for both Fermi liquids $I_{L}=0,-\pi / 2$. However, for $D \simeq D_{c}$ a singularity appears just on the Fermi level, being responsible of the non-trivial $I_{L}$. We conjeture that this singularity is related with the simultaneous creation, as $D \rightarrow D_{c}$ from below, of a zero and a pole of the impurity Green's function at the Fermi level, as it happens in an analogous way in other topological transitions in extended systems [34, 35].

In order to further characterize the critical region, the impurity contribution to the entropy as a function of temperature is plotted in Fig. 3. It can be clearly seen that, for $D$ close to $D_{c}$, there is a plateau at $S_{\mathrm{imp}}=\frac{1}{2} \ln (2)$, the fractional entropy usually associated with the $2 \mathrm{CK}$ physics [36]. Also, a shoulder at $S \simeq 3$ is noticeable, corresponding to the three-fold degeneracy of the $S=1$ impurity states at intermediate temperature. For other parameters (not shown in this work), this shoulder transforms in a clear plateau. At higher temperatures, out of the figure, there is a plateau at $S_{\mathrm{imp}}=7$ corresponding to the total number of localized impurity states in the model.

Another signature of $2 \mathrm{CK}$-like behavior close to $D_{c}$ is the fact that the NRG spectrum, as a function of the NRG iteration number $N$ (see supplemental material [30]), has an extended plateau for intermediate $N$, corresponding to a (unstable) fixed-point without the typical

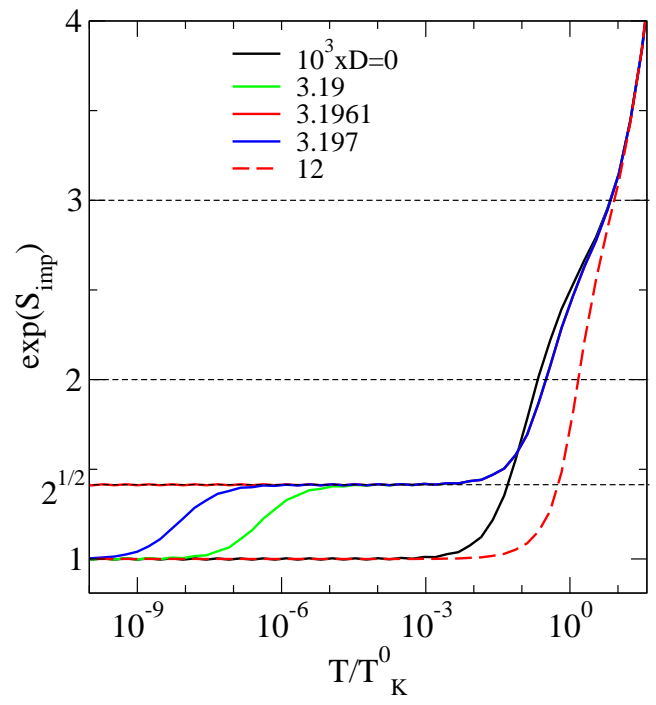

FIG. 3: (Color online) Impurity entropy as a function of temperature for several single-ion anisotropy $D$ close to the TQPT.

odd/even alternation and uniform level spacing of the conventional Kondo effect 37].

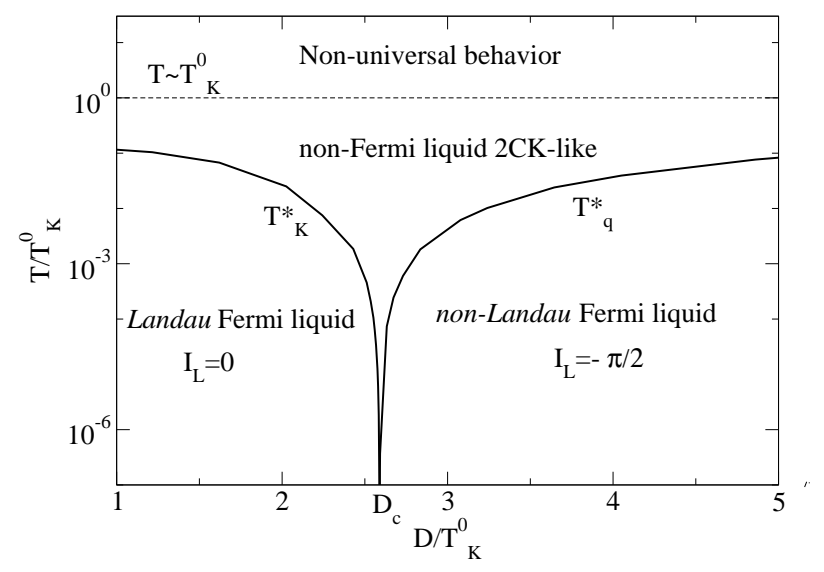

FIG. 4: Sketch of the phase diagram of the $S=1$ impurity model as a function of temperature and anisotropy. The solid lines indicate crossover regions between Fermi and non-Fermi liquid behaviors, while the dashed one signals the onset of non-universal behavior at higher temperatures.

Using the energy scales $T_{K}^{*}$ and $T_{q}^{*}$, we can summarize our findings in the phase diagram sketched in Fig. 4. with its "classical" Fermi liquid regions at both sides of the critical point, while the usual (non-Fermi liquid) quantum critical wedge emerges from the quantum critical point $D_{c}$ at zero temperature.

Summary- We have found a topological quantum phase transition between two Fermi liquids through an intermediate non-Fermi liquid 2CK-like phase in a simple model consisting in a $S=1$ impurity coupled to two conduc- 
tion bands, where the driving parameter of the transition is the single-ion magnetic anisotropy $D$. This model has experimental relevance for transport through nanostructures formed by Ni impurities in O-doped gold chains. The relative magnitude of anisotropy to the Kondo temperature $T_{K}^{0}$ can be experimentally tunable, rendering possible to observe the transition: $D / T_{K}^{0}$ can be modified by changing the effective $\varepsilon_{d}$ of the $\mathrm{Ni}$ atom by doping or it can be increased by mechanically streching the gold chains [2]. We expect that our work will stimulate further experimental work in similar systems. In particular, it would be interesting to find experimental probes that can distinguish between Fermi liquids characterized by different values of the topological invariant Luttinger integral.

Acknowledgements- We acknowledge financial support provided by PIP 112-201501-00506 of CONICET and PICT 2013-1045 of the ANPCyT.

[1] N. Roch, S. Florens, V. Bouchiat, W. Wernsdorfer, and F. Balestro, Quantum phase transition in a singlemolecule quantum dot, Nature (London) 453, 633 (2008).

[2] J. J. Parks, A. R. Champagne, T. A. Costi, W. W. Shum, A. N. Pasupathy, E. Neuscamman, S. Flores-Torres, P. S. Cornaglia, A. A. Aligia, C. A. Balseiro, G. K.-L. Chan, H. D. Abruña, and D. C. Ralph, Mechanical Control of Spin States in Spin-1 Molecules and the Underscreened Kondo Effect, Science 328, 1370 (2010).

[3] For a review see S. Florens, A, Freyn, N. Roch, W. Wernsdorfer, F. Balestro, P. Roura-Bas, and A. A. Aligia, Universal transport signatures in two-electron molecular quantum dots: gate-tunable Hund's rule, underscreened Kondo effect and quantum phase transitions, J. Phys. Condens. Matter 23, 243202 (2011); references therein.

[4] G. Katsaros, P. Spathis, M. Stoffel, F. Fournel, M. Mongillo, V. Bouchiat, F. Lefloch, A. Rastelli, O. G. Schmidt, and S. De Franceschi, Hybrid superconductorsemiconductor devices made from self-assembled SiGe nanocrystals on silicon, Nat. Nanotechnol. 5, 458 (2010).

[5] T. S. Jespersen, K. Grove-Rasmussen, J. Paaske, K. Muraki, T. Fujisawa, J. Nygard, and K. Flensberg, Gatedependent spinorbit coupling in multielectron carbon nanotubes, Nature Physics 7, 348 (2011).

[6] J. C. Oberg, M. R. Calvo, F. Delgado, M. Moro-Lagares, D. Serrate, D. Jacob, J. Fernandez-Rossier, and C. F. Hirjibehedin, Control of single-spin magnetic anisotropy by exchange coupling, Nat. Nanotechnol. 9, 64 (2013).

[7] B. W. Heinrich, L. Braun, J. I. Pascual, and K. J. Franke, Tuning the magnetic anisotropy of single molecules, Nano. Lett. 15, 4024 (2015).

[8] R. Hiraoka, E. Minamitani, R. Arafune, N. Tsukahara, S. Watanabe, M. Kawai, and N. Takagi, Single-molecule quantum dot as a Kondo simulator, Nature Commun. 8, 16012 (2017).

[9] H. Ohnishi, Y. Kondo, and K. Takayanagi, Quantized conductance through individual rows of suspended gold atoms, Nature 395, 780 (1998).

[10] V. Rodrigues, J. Bettini, P. C. Silva, and D. Ugarte, Evi- dence for Spontaneous Spin-Polarized Transport in Magnetic Nanowires Phys. Rev. Lett. 91, 096801 (2003).

[11] A. A. Aligia, K. Hallberg, C.D. Batista and G. Ortiz, Phase diagrams from topological transitions: The Hubbard chain with correlated hopping, Phys. Rev. B 61, 7883 (2000)

[12] A. A. Aligia A. Anfossi, L. Arrachea, C. Degli Esposti Boschi, A. O. Dobry, C. Gazza, A. Montorsi, F. Ortolani, and M. E. Torio, Incommmensurability and Unconventional Superconductor to Insulator Transition in the Hubbard Model with Bond-Charge Interaction, Phys. Rev. Lett. 99, 206401 (2007).

[13] L.-M. Duan, Effective Hamiltonian for Fermions in an Optical Lattice across a Feshbach Resonance, Phys. Rev. Lett. 95, 243202 (2005); General Hubbard model for strongly interacting fermions in an optical lattice and its phase detection, Europhys. Lett. 81, 20001 (2008).

[14] M. Fabrizio, A.O. Gogolin, and A.A. Nersesyan, From band insulator to Mott insulator in one dimension, Phys. Rev. Lett. 83, 2014 (1999).

[15] M. E. Torio, A. A. Aligia, and H. A. Ceccatto, Phase diagram of the Hubbard chain with two atoms per cell, Phys. Rev. B 64, 121105(R) (2001)

[16] A. A. Aligia, K. Hallberg, B. Normand, and A. P. Kampf, Detection of Topological Transitions by Transport Through Molecules and Nanodevices, Phys. Rev. Lett. 93, 076801 (2004).

[17] J. M. Luttinger, Fermi surface and some simple equilibrium properties of a system of interacting fermions, Phys. Rev. 119, 1153 (1960).

[18] J. M. Luttinger and J. C. Ward, Ground-state energy of a many-fermion system. II, Phys. Rev. 118, 1417 (1960).

[19] D. C. Langreth, Friedel Sum Rule for Anderson's Model of Localized Impurity States, Phys. Rev. 150, 516 (1966).

[20] A Yoshimori and A Zawadowski, Restricted Friedel sum rules and Korringa relations as consequences of conservation laws, J. Phys. C 15, 5241 (1982).

[21] The integral $I_{L}$ is in Eq. (63) of Ref. 18, Eq. (23) of Ref. 19, and Eq. (3.3) of Ref. 20. In our case it is given by Eq. (4).

[22] O. J. Curtin, Y. Nishikawa, A. C. Hewson, and D. J. G. Crow, Fermi liquids and the Luttinger theorem, J. Phys. Commun. 2, 031001 (2018).

[23] Y. Nishikawa, O. J. Curtin, A. C. Hewson, and D. J. G. Crow, Magnetic field induced quantum criticality and the Luttinger sum rule, arXiv:1712.08771.

[24] K. Seki and S. Yunoki, Topological interpretation of the Luttinger theorem, Phys. Rev. B 96, 085124 (2017).

[25] S. Di Napoli, M. A. Barral, P. Roura-Bas, L. O. Manuel, A. M. Llois, and A. A. Aligia, Kondo physics in a $\mathrm{Ni}$ impurity embedded in O-doped Au chains, Phys. Rev. B 92, 085120 (2015).

[26] M. A. Barral, S. Di Napoli, G. Blesio, P. Roura-Bas, A. Camjayi, L. O. Manuel, and A. A. Aligia, Kondo behavior and conductance through $3 d$ impurities in gold chains doped with oxygen, J. Chem. Phys. 146, 092315 (2017).

[27] W. H. A. Thijssen, D. Marjenburgh, R. H. Bremmer, and J. M. van Ruitenbeek, Oxygen-enhanced atomic chain formation, Phys. Rev. Lett. 96, 026806 (2006).

[28] S. Di Napoli, A. Thiess, S. Blügel, and Y. Mokrousov, Modelling impurity-assisted chain creation in noble-metal break junctions, J. Phys. Condens. Matter 24, 135501 (2012).

[29] Y. Nishikawa, D. J. G. Crow, and A. C. Hewson, Conver- 
gence of energy scales on the approach $t$ a local quantum critical point, Phys. Rev. Lett. 108, 056402 (2012).

[30] See Supplemental Material for details about the NRG calculations, the estimation of realistic parameters of the impurity model, results on the $S=1$ Kondo impurity coupled to two conduction bands, and the topological interpretation of the Luttinger integral.

[31] R. Žitko, NRG Ljubljana - open source numerical renormalization group code http://nrgljubljana.ijs.si (2014).

[32] P. S. Cornaglia, P. Roura Bas, A. A. Aligia and C. A. Balseiro, Quantum transport through a stretched spin-1 molecule, Europhys. Lett. 93, 47005 (2011)

[33] R. Žitko, private communication.

[34] S. Sakai, Y. Motome, and M. Imada, Evolution of elec- tronic structure of doped Mott insulators: Reconstruction of poles and zeros of Green's function, Phys. Rev. Lett. 102, 056404 (2009).

[35] V. Gurarie, Single-particle Green's functions and interacting topological insulators, Phys. Rev. B 83, 085426 (2011).

[36] A. K. Mitchell, E. Sela, and D. E. Logan, Two-channel Kondo physics in two-impurity Kondo models, Phys. Rev. Lett. 108, 086405 (2012).

[37] H. B. Pang and D. L. Cox, Stability of the fixed point of the two-channel Kondo Hamiltonian, Phys. Rev. B, 44, 9454 (1991). 


\section{Supplemental material for: Topological quantum phase transition between Fermi liquid phases in an Anderson impurity model}

We begin expressing the impurity Hamiltonian in terms of fermionic operators, by means of a two-orbital Anderson impurity model, in order to be solved with the "NRG Ljubljana" package. In Section II, we discuss the estimation of realistic parameters from ab-initio calculations for the Anderson impurity model. In Section III, we discuss some aspects of the NRG spectra. In Section IV, we present results for the $S=1$ Kondo impurity model with single-ion anisotropy $D$ and coupled to two degenerate conduction bands, that show that topological quantum phase transitions at finite $D_{c}$, as we have found in the Anderson model, seem to be generic for this kind of fully screened anisotropic $S=1$ impurity systems. Finally, in Section V, we give a topological interpretation of the Luttinger integral.

\section{TWO-ORBITAL ANDERSON IMPURITY MODEL USED IN NRG CALCULATIONS}

In order to use the "NRG Ljubljana" code [S1] for the resolution of Hamiltonian (1) -whose impurity degrees of freedom are expressed in terms of Hubbard operators-, we resort to a degenerate two-orbital Anderson impurity model, which involves only fermionic operators, and therefore it can be treated straightfowardly with the above mentioned code. The Anderson Hamiltonian is given by

$$
\begin{aligned}
H & =\sum_{\alpha \sigma} \varepsilon_{d} d_{\alpha \sigma}^{\dagger} d_{\alpha \sigma}+U \sum_{\alpha} n_{d \alpha \uparrow} n_{d \alpha \downarrow}+U^{\prime} n_{d_{x z}} n_{d_{y z}}- \\
& -J_{H} \vec{S}_{d_{x z}} \cdot \vec{S}_{d_{y z}}++J^{\prime}\left(d_{x z \uparrow}^{\dagger} d_{x z \downarrow}^{\dagger} d_{y z \downarrow} d_{y z \uparrow}+\text { H.c. }\right)+D S_{z}^{2}+ \\
& +\sum_{\nu k \alpha \sigma} \varepsilon_{k} c_{\nu k \alpha \sigma}^{\dagger} c_{\nu k \alpha \sigma}+\sum_{\nu k \alpha \sigma}\left(V_{\nu} c_{\nu k \alpha \sigma}^{\dagger} d_{\alpha \sigma}+\text { H.c. }\right)
\end{aligned}
$$

where $d_{\alpha \sigma}^{\dagger}$ creates a hole with energy $\varepsilon_{d}$ in the $3 d$ shell of the Ni atom with symmetry $\alpha=x z, y z$, while $c_{\nu k \alpha \sigma}^{\dagger}$ creates a hole in the $5 d_{\alpha}$ shell of the Au atom and $\nu=L, R$ denotes the left or the right side of the Ni atom, respectively. The energies $\varepsilon_{d}, \varepsilon_{k}$ are measured relative to the Fermi level, $\varepsilon_{F}=0 . U\left(U^{\prime}\right)$ is the intra- (inter-) orbital Coulomb repulsion, $J_{H}$ the ferromagnetic Hund exchange, $J^{\prime}$ the pair-hopping parameter, and $D$ the uniaxial magnetic anisotropy. The hopping $V_{\nu}$ characterizes the tunneling between the $\mathrm{Ni}$ and $\mathrm{Au}$ states at each side, and it enters the hybridization $\Delta=\pi \sum_{\nu k}\left|V_{\nu}\right|^{2} \delta\left(\omega-\varepsilon_{k}\right)$, assumed independent of energy.

To obtain the correct ground state configuration of the $\mathrm{Ni}$ atom -consisting of two holes in the degenerate $3 \mathrm{~d}_{x z}$, $3 \mathrm{~d}_{y z}$ orbitals Hund-coupled to spin $S=1$, but close to the mixed valence regime due to the fluctuations between $3 \mathrm{~d}^{8}$ and $3 \mathrm{~d}^{9}$ configurations [S2, S3] -, we have to project out some states. The configurations with an electron occupancy equals to 0, 3, and 4 are removed exactly in the used Hilbert space, while the singlet states with 2 electrons are projected out assigning them a very high energy. Thus, we take $U^{\prime}=J_{H} / 4$ and $U=J^{\prime}=J_{H}$, with $J_{H}$ large enough $\left(J_{H} \sim 1000 W, W=1\right.$ as the half-bandwidth of the conduction electrons) so that triplet and one-hole states remain the only relevant (low-energy) states, separated between them by an energy $\varepsilon_{d}$.

\section{ANDERSON IMPURITY MODEL PARAMETERS FOR NI ATOMS IN O-DOPED AU CHAINS}

LDA calculations give us access to some parameters of the Anderson Hamiltonian [S2]: conduction electron halfbandwidth, hybridization, and on-site energy $\varepsilon_{d}$. Also, LDA yields the hole occupancy $n_{d}$ of the $3 d$ orbitals. Of all these magnitudes, $\varepsilon_{d}$ is by far the least reliable quantity because, to some extent, the ab initio computed orbital energies include contributions from Coulomb repulsions at a mean-field level, that should not be present in the bare $\varepsilon_{d}$ of the Anderson model. Instead of taking the LDA $\varepsilon_{d}$ as the bare orbital energy, it is better to fix it by asking that the Anderson model and LDA occupancy numbers coincide [S4].

For $\mathrm{Ni}$ atoms in $\mathrm{O}$ doped $\mathrm{Au}$ chains, LDA estimations are $W=5 \mathrm{eV}, \Delta=0.115 \mathrm{eV}$, and $\varepsilon_{d}=-2 \Delta=-0.23$ $\mathrm{eV}$ [S2]. If we consider these values in the Anderson model, we obtain a NRG Kondo temperature that is very low, $T_{K}^{0} \simeq 0.1 \mathrm{meV}\left(D_{c} \simeq 0.45 \mathrm{meV}\right)$. This is understandable as the hole occupancy number, $n_{d}^{N R G}=1.943$, indicates that, for this on-site energy, the impurity is well inside of the Kondo regime (this implies a low $T_{K}$ ), while, on the other hand, the hole occupancy obtained with LDA $\left(n_{d}=1.44\right.$, taken from Table I, first row of Ref. S2) corresponds to a regime close to mixed valence. So, this a clear indication that the LDA estimated $\varepsilon_{d}$ is not a good input for the 
Anderson impurity Hamiltonian, as its value is well below the Fermi level. Furthermore, the Haldane shift of $\varepsilon_{d}$, in the $S=1$ case, is given by [S5]

$$
\varepsilon_{d}^{*}=\varepsilon_{d}-\frac{\Delta}{2 \pi} \log \frac{W}{\Delta},
$$

and it pushes the bare on-site energy to even lower values.

To get the same hole occupancy as in the LDA calculations, we need to take a positive $\varepsilon_{d} \simeq 0.93 \Delta$, leaving the other parameters fixed, in the Anderson impurity model. For this $\varepsilon_{d}$, the Kondo temperature is $T_{K}^{0}=6$ meV, so the critical anisotropy $D_{c} \sim 15 \mathrm{meV}$, above the estimated $D(\simeq 8 \mathrm{meV})$ of Ni atoms in O-doped gold chains. Thus, it is possible to promote the topological quantum phase transition reducing the Kondo temperature $T_{K}^{0}$ by means of the mechanical stretching of the gold chains.

In our work, in order to highlight the non-Fermi liquid region, we consider an intermediate relative value of the on-site energy, $\varepsilon_{d}=-\Delta / 5$. The corresponding hole occupancy, $n_{d}=1.576$, is a bit above the value given by LDA, however the impurity is in the crossover region between Kondo and mixed valence regimes, as infered from LDA results for the occupancies. For any parameter set choice that corresponds to the Kondo regime or that crossover region, the qualitative impurity behavior remains the same.

\section{NRG ENERGY SPECTRUM FOR THE ANDERSON IMPURITY MODEL}

The behavior of the low-energy eigenvalues of the NRG Hamiltonians $H_{N}$ with the iteration number $N$ gives important information about the fixed points of an impurity system [S6]. In Fig. S1, the NRG eigenvalues $\left(E_{N}\right)$ of lower energy as a function of the length of the Wilson chain $N$ are shown, for an anisotropy close to $D_{c}$ in the Anderson model (same parameters as in the main paper). At intermediate $N(15 \lesssim N \lesssim 55)$ there is an extended plateau signalling the existence of an unstable fixed point at intermediate energies or temperatures, in complete agreement with the non-Fermi 2CK fingerprints we have found in the impurity entropy, conductance, and spectral function. To strengthen this point, two features can be seen in Fig. S1 (and at higher $E_{N}$ not shown in the figure): there is no even/odd alternation in the spectra, and the energy level spacing is not uniform, both are known results for the $2 \mathrm{CK}$ state due to its non-Fermi liquid nature [S7].

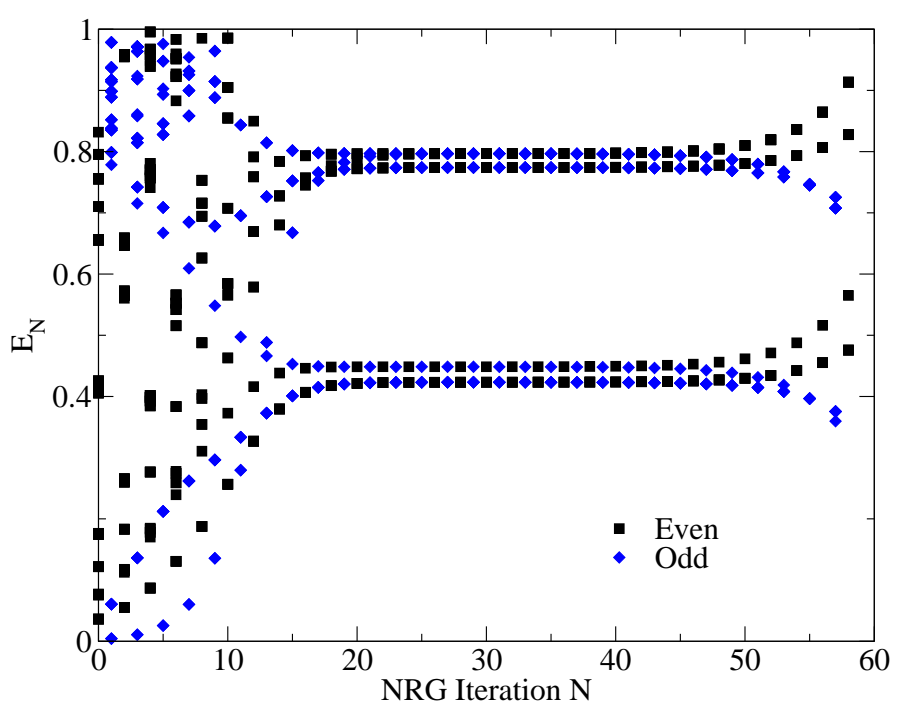

FIG. S1: Flow diagram of the eigenvalues obtained by NRG as a function of the iteration number for $D=0.0031961 \simeq D_{c}$. Different symbols are used for even and odd iteration numbers. The ground state energy is set to zero. Other parameters as in the main paper.

We consider as quantum numbers of the NRG eigenvalues, $Q$-the difference of the number of electrons with respect to the ground state- and the spin projection $S_{z}$. The low-energy eigenvalues, beginning with the ground state, have quantum numbers $\left(Q=0, S_{z}=0\right),\left(Q=-1, S_{z}= \pm 1 / 2\right),\left(Q=-2, S_{z}=0\right),\left(Q=1, S_{z}= \pm 1 / 2\right)$, $\left(Q=0, S_{z}=-1,3 / 2\right)$. We were not able to match one to one the NRG eigenvalues of our critical $S=1$ Anderson 
impurity model and the known eigenvalues of the $S=1 / 2$ two-channel Kondo model [S8] , probably due to the presence of marginal operators.

\section{$S=1$ KONDO IMPURITY COUPLED TO TWO CONDUCTION BANDS}

In order to check that our results are generic for a magnetic impurity with two orbitals hybridized with two conduction bands, at or close to the $n_{\mathrm{imp}} \simeq 2$ Kondo regime, we have performed a NRG study of the $S=1$ Kondo impurity model coupled with two conduction bands,

$$
H=\sum_{\mathbf{k} \nu \alpha \sigma} \varepsilon_{\mathbf{k} \nu \alpha} c_{\mathbf{k} \nu \alpha \sigma}^{\dagger} c_{\mathbf{k} \nu \alpha \sigma}+\sum_{\nu \alpha} J_{\nu \alpha} \mathbf{S}_{\mathrm{imp}} \cdot \mathbf{s}_{\alpha \nu}+D\left(S_{\mathrm{imp}}^{z}\right)^{2}
$$

where, as in the Anderson model, $\nu=L, R$ refers to the left and right leads connected with the impurity, $\alpha=x z, y z$ refers to the two degenerate conduction electron bands, $\mathbf{S}_{\mathrm{imp}}$ is the $S=1$ spin operator of the impurity, $\mathbf{s}_{\nu \alpha}$ is the conduction electron spin density at the impurity site, and $D$ is the single-ion magnetic anisotropy. In what follows, we consider an equal Kondo exchange coupling of the impurity to both leads and both conduction bands, $J_{\nu \alpha}=J$, and take $J=0.2$, being the half-bandwidth of the degenerate conduction bands the unit of energy $(W=1)$. We use discretization parameters $\Lambda=2$ and 3, keeping up to 4000 NRG states, and the results are $z$-averaged with $N_{z}=4$.

The calculated Kondo temperature for $D=0$ is $T_{K}^{0}=4.2 \times 10^{-5}(\Lambda=3)$. We find again a quantum phase transition between fully-screened Kondo and anisotropy-quenched impurity spin phases at a finite value of the anisotropy, $D_{c}=1.352 \times 10^{-4}$. Notice that the relation $D / T_{K}^{0}=3.21$ is close to the corresponding one in the Anderson model.

In the left panel of Fig. S2, the differential conductance per channel as a function of $T / T_{K}^{0}$ is shown for different $D$ across the quantum phase transition. The behavior of $G$ with $D$ is the same as in the Anderson model. The only difference is that now the conductance (neglecting small numerical inacurracies) reaches the unitary limit at small temperatures $G_{\alpha}(T \rightarrow 0)=G_{0}$ for $D<D_{c}$, while it goes to zero for $D>D_{c}$. This has to do with the fact that the Kondo impurity model has particle-hole symmetry. In the right panel of Fig. S2, the impurity contribution to the entropy is plotted as a function of $T / T_{K}^{0}$ for the same anisotropies as in the conductance figure. Again, the result is qualitatively the same as for the Anderson model. Thus, the picture found in the main paper holds for the $S=1$ Kondo impurity: at $D_{c}$ there is a quantum phase transition between two Fermi liquids, and in the critical region there is a non-Fermi liquid phase with characteristics proper of the two-channel Kondo effect.
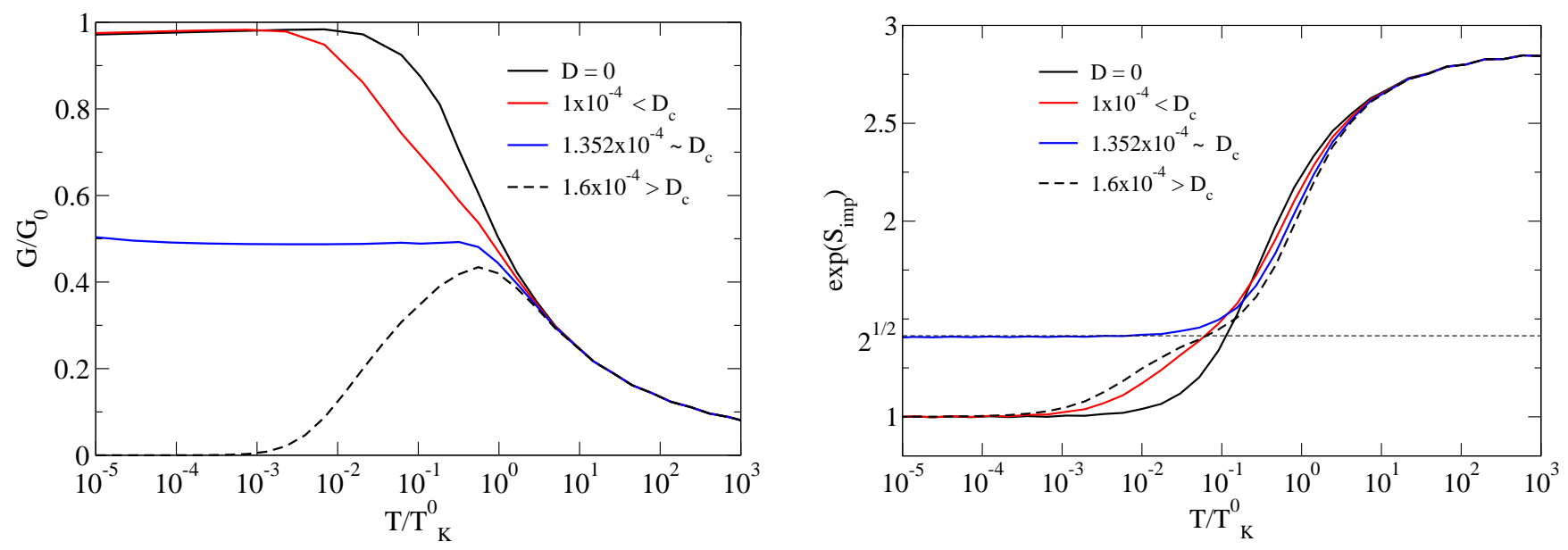

FIG. S2: (Left) Electrical differential conductance as a function of $T / T_{K}^{0}$ for several values of the single-ion anisotropy $D$. (Right) Impurity entropy contribution for the $S=1$ Kondo model as a function of temperature, for several values of $D$.

A function $\rho_{\alpha}(\omega)$ equivalent to the impurity spectral function can be defined for the Kondo model [S9]. This spectral function is defined through the $t$-matrix of the conduction electrons using the relation $\rho_{\alpha}(\omega)=-\pi \rho_{\alpha}^{0} \operatorname{Im} t_{\alpha}(\omega)$, where $\rho_{\alpha}^{0}=1 / 2 W$ is the conduction electron density of states, that is assumed energy independent. $t_{\alpha}(\omega)$ is the $t$-matrix 
for the conduction electrons with symmetry $\alpha$ scattering off the magnetic impurity, and it is defined by the relation

$$
G_{\alpha, \mathbf{k k}^{\prime}}(\omega)=G_{\alpha, \mathbf{k}}^{0}(\omega) \delta_{\mathbf{k k}^{\prime}}+G_{\alpha, \mathbf{k}}^{0}(\omega) t_{\alpha}(\omega) G_{\alpha, \mathbf{k}^{\prime}}^{0}(\omega),
$$

where $G_{\alpha \mathbf{k}}^{0}=1 /\left(\omega+i 0^{+}-\varepsilon_{\mathbf{k}}\right)$ is the Green's function for the conduction band with symmetry $\alpha$ in the abscence of the impurity. Fig. S3 shows $\rho_{\alpha}(\omega)$ as a function of $\omega / T_{K}^{0}$ for several values of $D[$ S10] Again, the same behavior as in the Anderson model is recovered. However, thanks to the particle-hole symmetry of the Kondo model around the Fermi level $\omega=0$, now it is clearer that for $D \rightarrow D_{c}$ and $\omega \rightarrow 0, \rho_{\alpha}(\omega)$ takes half of its value in the fully screened Kondo phase. This is another characteristic of the non-Fermi-liquid two-channel Kondo effect.

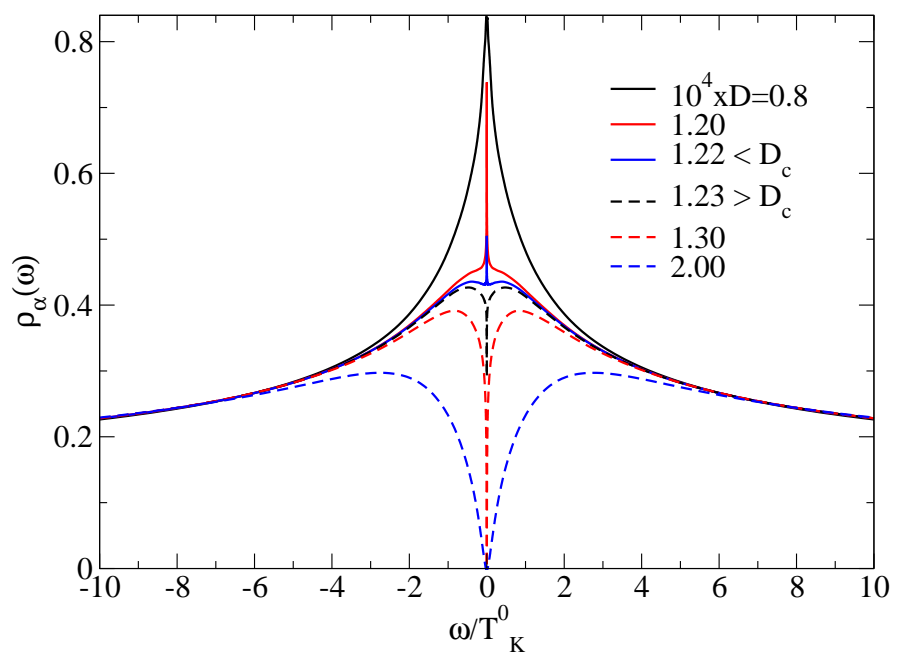

FIG. S3: Spectral function $\rho_{\alpha}(\omega)$ versus $\omega / T_{K}^{0}$ for several values of the single-ion anisotropy $D$ for the $S=1$ Kondo impurity model.

\section{TOPOLOGICAL INTERPRETATION OF THE LUTTINGER INTEGRAL}

In this section, we present a topological interpretation of the Luttinger integral for the Anderson impurity model, following the guidelines of the recent work by Seki and Yunoki [S11].

We rewrite Hamiltonian (S1) as $H=H_{0}+H_{\text {int }}$, with the non-interacting part

$$
H_{0}=\sum_{\nu k \alpha \sigma} \varepsilon_{k} c_{\nu k \alpha \sigma}^{\dagger} c_{\nu k \alpha \sigma}+\sum_{\nu k \alpha \sigma}\left(V_{\nu} c_{\nu k \alpha \sigma}^{\dagger} d_{\alpha \sigma}+\text { H.c. }\right)+\sum_{\alpha \sigma} \tilde{\varepsilon}_{d} d_{\alpha \sigma}^{\dagger} d_{\alpha \sigma},
$$

where $\tilde{\varepsilon}_{d}$ is an effective $d$ level determined selfconsistenly, so that the total non-interacting occupancy $N$ is the same as the interacting one (for example, in the symmetric single-orbital Anderson model where $\varepsilon_{d}=-U / 2, \tilde{\varepsilon}_{d}=0$ ). $H_{\text {int }}=H-H_{0}$ includes all the two-body terms. We express the single-particle Green's function as an $L_{s} \times L_{s}$ matrix $\mathbf{G}(z)$, where $L_{s}$ is the number of single-particle states and $z$ is the complex frequency. The average electron number $N$ is given by the contour integral

$$
N=\oint_{\Gamma} \frac{d z}{2 \pi i} n_{F}(z) \operatorname{tr} \mathbf{G}(z),
$$

where the contour $\Gamma$ encloses the singularities of $\mathbf{G}(z)$ (all in the real-frequency axis) in the counterclockwise direction, as shown in Fig. 2(a) of Ref. S11, and $n_{F}$ is the Fermi function. The trace can be expressed as

$$
\operatorname{tr} \mathbf{G}(z)=\sum_{k \nu \alpha \sigma} G_{k \nu \alpha \sigma}(z)+\sum_{\alpha \sigma} G_{d \alpha \sigma}(z),
$$

where $G_{k \nu \alpha \sigma}$ and $G_{d \alpha \sigma}$ are the diagonal elements of $\mathbf{G}(z)$. In the following, we explicitly consider that the Green's functions do not depend on the orbital and spin indices. By the equation-of-motion method, we can obtain the relation

$$
G_{k \nu}(z)=\frac{1}{z-\varepsilon_{k}}+\left|V_{\nu}\right|^{2}\left(\frac{1}{z-\varepsilon_{k}}\right)^{2} G_{d}(z)
$$


what brings us to the expression

$$
\operatorname{tr} \mathbf{G}(z)=\sum_{k \nu \alpha \sigma} \frac{1}{z-\varepsilon_{k}}+\sum_{\alpha \sigma}\left(1-\frac{\partial \Gamma(z)}{\partial z}\right) G_{d}(z)
$$

where $\Gamma(z)=\sum_{k \nu}\left|V_{\nu}\right|^{2} \frac{1}{z-\varepsilon_{k}}$ is the complex hybridization function, whose imaginary part is $\Delta$.

Using the Dyson equation,

$$
G_{d}(z)=\frac{1}{z-\tilde{\varepsilon}_{d}-\Gamma(z)-\Sigma_{d}(z)}
$$

where $\Sigma_{d}(z)$ is the impurity self-energy due to $H_{\text {int }}$, (S9) can be rewritten as

$$
\operatorname{tr} \mathbf{G}(z)=\sum_{k \nu \alpha \sigma} \frac{1}{z-\varepsilon_{k}}+\sum_{\alpha \sigma} \frac{\partial \log \left(G_{d}\right)^{-1}}{\partial z}+\sum_{\alpha \sigma} \frac{\partial \Sigma_{d}(z)}{\partial z} G_{d}(z) .
$$

As we turn off the interactions $\left(H_{\mathrm{int}}=0\right)$, the electron number is given also by the contour integral

$$
N=\oint_{\Gamma} \frac{d z}{2 \pi i} n_{F}(z) \operatorname{tr} \mathbf{G}^{0}(z)
$$

where $\mathbf{G}^{0}(z)$ is the single-particle Green's function corresponding to $H=H_{0}$. If we equal (즈) and (S12), using the expression (S11) (and taking into account that $\Sigma_{d \alpha \sigma}=0$ for $H_{\text {int }}=0$ ), we get

$$
\oint_{\Gamma} \frac{d z}{2 \pi i} n_{F}(z) \frac{\partial \log D_{d}(z)}{\partial z}=-\oint_{\Gamma} \frac{d z}{2 \pi i} n_{F}(z) \frac{\partial \Sigma_{d}(z)}{\partial z} G_{d}(z)
$$

where $D_{d}(z)=G_{d}^{0}(z) / G_{d}(z)$. Eq. (S13) corresponds to Eqs. (51) and (53) of Ref. S11 for the Anderson impurity Hamiltonian. The contour integral in its left-hand side is associated with the deviation of the Luttinger volume from the non-interacting one due to many-body interactions in extended systems.

If $F(z)$ is an analytical function in the complex plane except, perhaps, on the real axis, the following relation holds:

$$
\oint_{\Gamma} \frac{d z}{2 \pi i} n_{F}(z) F(z)=-\frac{1}{\pi} \operatorname{Im} \int_{-\infty}^{\infty} n_{F}(\omega) F(\omega+i \eta) d \omega
$$

where $\eta$ a positive infinitesimal. Using this relation and Eq. (S13), the Luttinger integral (4) can be written as

$$
I_{L}=-\pi \lim _{T \rightarrow 0} \oint_{\Gamma} \frac{d z}{2 \pi i} n_{F}(z) \frac{\partial \Sigma_{d}(z)}{\partial z} G_{d}(z)=\pi \lim _{T \rightarrow 0} \oint_{\Gamma} \frac{d z}{2 \pi i} n_{F}(z) \frac{\partial \log D_{d}(z)}{\partial z} .
$$

At zero temperature, the contour integral $\Gamma$ is reduced to the contours $\Gamma_{<}$and $\Gamma_{0}$, which enclose the negative real axis and the origin, respectively (see Fig. 2(b) in Ref. S11), and

$$
\lim _{T \rightarrow 0} \oint_{\Gamma} \frac{d z}{2 \pi i} n_{F}(z) \frac{\partial \log D_{d}(z)}{\partial z}=n_{D_{d}}\left(\Gamma_{<}\right)+\frac{1}{2} n_{D_{d}}\left(\Gamma_{0}\right)
$$

where, for a contour $\mathcal{C}$ in the complex $z$ plane, $n_{D_{d}}(\mathcal{C})$ is the winding number that counts the (signed-)number of times that the contour $D_{d}(\mathcal{C})$ encloses the origin of complex $D_{d}$ plane.

Consequently, the Luttinger integral,

$$
I_{L}=\pi n_{D_{d}}\left(\Gamma_{<}\right)+\frac{\pi}{2} n_{D_{d}}\left(\Gamma_{0}\right)
$$

has a clear topological character. In Ref. S11, the relation between these winding numbers and the singularities (poles and zeros) of the single-particle Greens function is stressed. 


\section{Luttinger integral for the Anderson atom}

In order to illustrate how the analytical structure of the Green's function may change with a varying Hamiltonian parameter, and its relation with the Luttinger integral, we analyze the evolution with $U$ of the Anderson impurity model in the atomic limit. The Hamiltonian is

$$
\hat{H}_{\mathrm{at}}=\varepsilon_{d} \hat{n}_{d}+U \hat{n}_{d \uparrow} \hat{n}_{d \downarrow}
$$

The impurity Green's function can be exactly calculated using the equation-of-motion method, and it is given by

$$
G_{d \sigma}(z)=\left(1-\frac{n_{d}}{2}\right) \frac{1}{z-\varepsilon_{d}}+\frac{n_{d}}{2} \frac{1}{z-\left(\varepsilon_{d}+U\right)},
$$

where $n_{d}$ is the impurity occupancy. The Dyson equation allows us to express the Green's function in terms of the impurity self-energy

$$
G_{d \sigma}(z)=\frac{1}{z-\varepsilon_{d}-\Sigma_{d \sigma}(z)}
$$

where

$$
\Sigma_{d \sigma}(z)=\frac{n_{d}}{2} U+\frac{\left(1-\frac{n_{d}}{2}\right) \frac{n_{d}}{2} U^{2}}{z-\varepsilon_{d}-\left(1-\frac{n_{d}}{2}\right) U} .
$$

If $\varepsilon_{d}<0$ and $\varepsilon_{d}+U<0$, then the electron occupancy is given by $n_{d}=2$ and

$$
G_{d \sigma}(z)=\frac{1}{z-\left(\varepsilon_{d}+U\right)}, \quad \Sigma_{d \sigma}(z)=U .
$$

Trivially, the Luttinger integral vanishes in this case. On the other hand, we take the non-interacting Green's function $G_{d \sigma}^{0}(z)=1 /\left(z-\varepsilon_{d}\right)$ (which gives the same interacting occupancy $n_{d}=1$ ), and, consequently,

$$
D_{d}(z)=\frac{G_{d \sigma}^{0}(z)}{G_{d \sigma}(z)}=\frac{z-\left(\varepsilon_{d}+U\right)}{z-\varepsilon_{d}} .
$$

As

$$
\frac{\partial \log D_{d}(z)}{\partial z}=\frac{1}{z-\left(\varepsilon_{d}+U\right)}-\frac{1}{z-\varepsilon_{d}}
$$

the winding number of $D_{d}(z)$ vanishes.

For $\varepsilon_{d}<0$ and $\varepsilon_{d}+U>0$, the electron occupancy is $n_{d}=1$, yielding

$$
G_{d \sigma}(z)=\frac{1}{2}\left[\frac{1}{z-\varepsilon_{d}}+\frac{1}{z-\left(\varepsilon_{d}+U\right)}\right], \quad \Sigma_{d \sigma}(z)=\frac{U}{2}+\left(\frac{U}{2}\right)^{2} \frac{1}{z-\left(\varepsilon_{d}+\frac{U}{2}\right)} .
$$

Notice that, in this case, the Green's function adquires simultaneously a new pole $\left(z_{p}=\varepsilon_{d}\right)$ and a zero $\left(z_{o}=\varepsilon_{d}+U / 2\right)$. The last one can be seen as a singularity of the self-energy.

As

$$
G_{d \sigma}(z) \frac{\partial \Sigma_{d \sigma}(z)}{\partial z}=\frac{1}{z-\left(\varepsilon_{d}+\frac{U}{2}\right)}-\frac{1}{2}\left[\frac{1}{z-\varepsilon_{d}}+\frac{1}{z-\left(\varepsilon_{d}+U\right)}\right],
$$

the Luttinger integral $I_{L}=-\pi / 2(+\pi / 2)$ if $\varepsilon_{d}$ is below (above) the symmetric point, $\varepsilon_{d}=-U / 2$. In order to get a non-interacting limit with the same occupancy, $n_{d}=1$, we need to shift $\varepsilon_{d}$ to the Fermi level $\left(\tilde{\varepsilon}_{d}=0\right)$, so $G_{d \sigma}^{0}(z)=1 / z$. As

$$
D_{d}(z)=\frac{G_{d \sigma}^{0}(z)}{G_{d \sigma}(z)}=\frac{\left(z-\varepsilon_{d}\right)\left(z-\left(\varepsilon_{d}+U\right)\right)}{z\left(z-\left(\varepsilon_{d}+\frac{U}{2}\right)\right)},
$$


the countour integral (S16) is $-1 / 2(+1 / 2)$ if $\varepsilon_{d}$ is below (above) the symmetric point, in agreement with the Luttinger integral values given above.

[S1] R. Žitko, NRG Ljubljana - open source numerical renormalization group code http://nrgljubljana.ijs.si (2014).

[S2] S. Di Napoli, M. A. Barral, P. Roura-Bas, L. O. Manuel, A. M. Llois, and A. A. Aligia, Kondo physics in a Ni impurity embedded in O-doped Au chains, Phys. Rev. B 92, 085120 (2015).

[S3] M. A. Barral, S. Di Napoli, G. Blesio, P. Roura-Bas, A. Camjayi, L. O. Manuel, and A. A. Aligia, Kondo behavior and conductance through $3 d$ impurities in gold chains doped with oxygen, J. Chem. Phys. 146, 092315 (2017).

[S4] M. Núñez, D. C. Freitas, F. Gay, J. Marcus, P. Strobel, A. A. Aligia, and M. Núñez-Regueiro, Orbital Kondo effect in $V$-doped 1T-CrSe $e_{2}$, Phys. Rev. B 88, 245129 (2013)

[S5] G. G. Blesio, L. O. Manuel, A. A. Aligia, and P. Roura-Bas, unpublished.

[S6] A. C. Hewson, The Kondo Problem to Heavy Fermions, (Cambridge University Press, Cambridge, England, 1997), ISBN 9780521599474.

[S7] H. B. Pang and D. L. Cox, Stability of the fixed point of the two-channel Kondo Hamiltonian, Phys. Rev. B, 44, 9454 (1991).

[S8] A. K. Mitchell, E. Sela, and D. E. Logan, Two-channel Kondo physics in two-impurity Kondo models, Phys. Rev. Lett. 108, 086405 (2012).

[S9] A. K. Mitchell and D. E. Logan, Two-channel Kondo phases and frustration-induced transitions in triple quantum dots, Phys. Rev. B 81, 075126 (2010).

[S10] For the computation of the spectral function $\rho_{\alpha}$, we take $\Lambda=2$ and we keep 3000 NRG states. As a consequence, $D_{c}=1.225 \times 10^{-4}$ and $T_{K}^{0}=3.8 \times 10^{-5}$ are a little different from the corresponding values that appear in the conductance and impurity entropy in Fig. $\mathbf{S 2}\left(D_{c}=1.352 \times 10^{-4}, T_{K}^{0}=4.2 \times 10^{-5}\right)$.

[S11] K. Seki and S. Yunoki, Topological interpretation of the Luttinger theorem, Phys. Rev. B 96, 085124 (2017). 